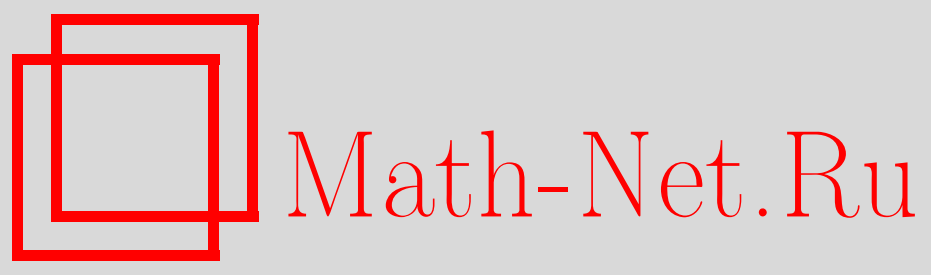

М. Ю. Балахнев, Формулы суперпозиции для интегрируемых векторных эволюционных уравнений, $T M \Phi$, 2008, том 154, номер 2, 261-267

DOI: https://doi.org/10.4213/tmf6167

Использование Общероссийского математического портала Math-Net.Ru подразумевает, что вы прочитали и согласны с пользовательским соглашением http://www . mathnet.ru/rus/agreement

Параметры загрузки:

IP : 54.162 .127 .20

26 апреля 2023 г., 17:41:06

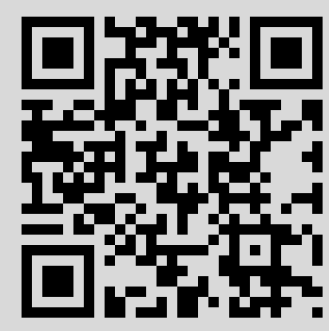




\title{
ФОРМУЛЫ СУПЕРПОЗИЦИИ ДЛЯ ИНТЕГРИРУЕМЫХ ВЕКТОРНЫХ ЭВОЛЮЦИОННЫХ УРАВНЕНИЙ
}

\author{
С использованием преобразования Беклунда построены формулы суперпо- \\ зиции для решений некоторых интегрируемых векторных уравнений.
}

Ключевые слова: интегрируемые векторные эволюционные уравнения, преобразования Беклунда, диаграмма Бианки.

\section{ВВЕДЕНИЕ}

В работе [1] была предложена бескомпонентная версия симметрийного подхода к классификации векторных уравнений и получен полный список интегрируемых уравнений на сфере $\mathbb{S}^{n}$. Классификация векторных уравнений специального вида приведена в работах [2], [3]. В работе [4] рассмотрены интегрируемые векторные уравнения на $\mathbb{S}^{n}$ более общего, чем в [1], вида. Таким образом, на сегодняшний день известно достаточно большое число интегрируемых векторных уравнений. Однако формула суперпозиции была получена только для решений векторных обобщений уравнения Шварца-КдФ [2] и мКдФ [5]. В нашей работе построены формулы суперпозиции для других наиболее простых и, на наш взгляд, интересных уравнений.

\section{1. ТЕХНИКА ВЫЧИСЛЕНИЙ}

Вывод формулы суперпозиции продемонстрируем на примере простейшего интегрируемого на сфере $\mathbb{S}^{n}$ уравнения

$$
\mathbf{z}_{t}=\mathbf{z}_{x x x}+3 \mathbf{z}_{x}^{2} \mathbf{z}_{x}+3\left(\mathbf{z}_{x}, \mathbf{z}_{x x}\right) \mathbf{z}, \quad \mathbf{z}^{2}=(\mathbf{z}, \mathbf{z})=1
$$

где $\mathbf{z}(t, x)$ есть вектор $n$-мерного векторного пространства $V$ со скалярным произведением $(\cdot, \cdot)$. Автопреобразование Беклунда для (1.1) было получено в [1] и записывается так:

$$
\mathbf{y}_{x}=\mathbf{z}_{x}-\left(\mathbf{y}, \mathbf{z}_{x}\right) \mathbf{y}-2\left(\mu-\frac{\left(\mathbf{y}, \mathbf{z}_{x}\right)}{(\mathbf{y}-\mathbf{z})^{2}}\right)(\mathbf{z}-(\mathbf{y}, \mathbf{z}) \mathbf{y}) .
$$

* Орловский государственный технический университет, Орел, Россия. Email: balakhnev@yandex.ru 
Если возвести равенство (1.2) в квадрат, найти из полученного выражения скалярное произведение $\left(\mathbf{y}, \mathbf{z}_{x}\right)$, а затем подставить его в (1.2), то преобразование (1.2) примет вид

$$
\mathbf{y}_{x}=\mathbf{z}_{x}-\frac{\mu(\mathbf{y}-\mathbf{z})^{2}}{2}(\mathbf{y}+\mathbf{z})+\frac{\mathbf{y}_{x}^{2}-\mathbf{z}_{x}^{2}}{2 \mu(\mathbf{y}-\mathbf{z})^{2}}(\mathbf{y}-\mathbf{z}) .
$$

Следуя Бианки [6], предположим коммутативность диаграммы

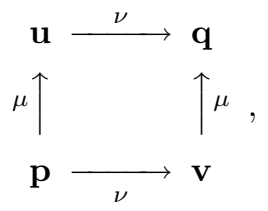

где $\mathbf{p}, \mathbf{u}, \mathbf{v}, \mathbf{q}$ - решения уравнения (1.1), связанные в соответствии с диаграммой преобразованиями Беклунда вида (1.3).

Из коммутативности диаграммы (1.4) следуют четыре векторных соотношения

$$
\begin{aligned}
& \mathbf{u}_{x}=\mathbf{p}_{x}-\frac{\mu(\mathbf{u}-\mathbf{p})^{2}}{2}(\mathbf{u}+\mathbf{p})+\frac{\mathbf{u}_{x}^{2}-\mathbf{p}_{x}^{2}}{2 \mu(\mathbf{u}-\mathbf{p})^{2}}(\mathbf{u}-\mathbf{p}), \\
& \mathbf{v}_{x}=\mathbf{p}_{x}-\frac{\nu(\mathbf{v}-\mathbf{p})^{2}}{2}(\mathbf{v}+\mathbf{p})+\frac{\mathbf{v}_{x}^{2}-\mathbf{p}_{x}^{2}}{2 \nu(\mathbf{v}-\mathbf{p})^{2}}(\mathbf{v}-\mathbf{p}), \\
& \mathbf{q}_{x}=\mathbf{u}_{x}-\frac{\nu(\mathbf{q}-\mathbf{u})^{2}}{2}(\mathbf{q}+\mathbf{u})+\frac{\mathbf{q}_{x}^{2}-\mathbf{u}_{x}^{2}}{2 \nu(\mathbf{q}-\mathbf{u})^{2}}(\mathbf{q}-\mathbf{u}), \\
& \mathbf{q}_{x}=\mathbf{v}_{x}-\frac{\mu(\mathbf{q}-\mathbf{v})^{2}}{2}(\mathbf{q}+\mathbf{v})+\frac{\mathbf{q}_{x}^{2}-\mathbf{v}_{x}^{2}}{2 \mu(\mathbf{q}-\mathbf{v})^{2}}(\mathbf{q}-\mathbf{v}) .
\end{aligned}
$$

Используя их, нетрудно получить следующее равенство:

$$
\begin{aligned}
& \frac{\nu(\mathbf{v}-\mathbf{p})^{2}}{2}(\mathbf{v}+\mathbf{p})-\frac{\mathbf{v}_{x}^{2}-\mathbf{p}_{x}^{2}}{2 \nu(\mathbf{v}-\mathbf{p})^{2}}(\mathbf{v}-\mathbf{p})-\frac{\mu(\mathbf{u}-\mathbf{p})^{2}}{2}(\mathbf{u}+\mathbf{p})+\frac{\mathbf{u}_{x}^{2}-\mathbf{p}_{x}^{2}}{2 \mu(\mathbf{u}-\mathbf{p})^{2}}(\mathbf{u}-\mathbf{p})= \\
& \quad=\frac{\nu(\mathbf{q}-\mathbf{u})^{2}}{2}(\mathbf{q}+\mathbf{u})-\frac{\mathbf{q}_{x}^{2}-\mathbf{u}_{x}^{2}}{2 \nu(\mathbf{q}-\mathbf{u})^{2}}(\mathbf{q}-\mathbf{u})-\frac{\mu(\mathbf{q}-\mathbf{v})^{2}}{2}(\mathbf{q}+\mathbf{v})+\frac{\mathbf{q}_{x}^{2}-\mathbf{v}_{x}^{2}}{2 \mu(\mathbf{q}-\mathbf{v})^{2}}(\mathbf{q}-\mathbf{v})
\end{aligned}
$$

Умножая это равенство скалярно на векторы $\mathbf{p}, \mathbf{u}, \mathbf{v}, \mathbf{q}$, мы получаем четыре уравнения. Из полученной системы выражаем величины $\mathbf{q}_{x}^{2},(\mathbf{q}, \mathbf{u}),(\mathbf{q}, \mathbf{v})$ и, подставив их в исходное уравнение, находим формулу суперпозиции для решений (1.1):

$$
\mathbf{q}=\zeta^{-1}(\mathbf{u}-\mathbf{v})^{2}\left(\xi \eta \mathbf{p}+(\mu+\nu)\left(\xi(\mathbf{v}-\mathbf{p})^{2} \mathbf{u}+\eta(\mathbf{u}-\mathbf{p})^{2} \mathbf{v}\right)\right)
$$

где

$$
\begin{gathered}
\xi=2 \nu(\mathbf{u}-\mathbf{p}, \mathbf{v})-\mu(\mathbf{u}-\mathbf{p})^{2}, \quad \eta=2 \mu(\mathbf{u}, \mathbf{v}-\mathbf{p})-\nu(\mathbf{v}-\mathbf{p})^{2} \\
\zeta=\left[2(\mathbf{u}+\mathbf{v}, \mathbf{p})-(\mathbf{u}+\mathbf{v})^{2}\right] \xi \eta-(\mathbf{v}-\mathbf{p})^{2} \xi^{2}-(\mathbf{u}-\mathbf{p})^{2} \eta^{2}
\end{gathered}
$$

Проведение расчетов при решении уравнения (1.1) облегчается как видом уравнения, так и тем, что оно интегрируемо на $\mathbb{S}^{n}$. Это означает, что для любого решения $\mathbf{u}$ мы имеем соотношение $\mathbf{u}^{2}=1$ и все его следствия. Кроме того, существует преобразование Беклунда для (1.1), зависящее от квазилокальных переменных. Можно проверить, что уравнение

$$
\tilde{y}-\tilde{z}=\mu(\mathbf{y}-\mathbf{z})^{2}
$$


совместно с (1.1) и (1.2); здесь $\tilde{y}=D_{x}^{-1}\left(\mathbf{y}_{x}, \mathbf{y}_{x}\right), \tilde{z}=D_{x}^{-1}\left(\mathbf{z}_{x}, \mathbf{z}_{x}\right)$ - квазилокальные переменные. Тогда преобразование (1.2) можно переписать в виде

$$
\mathbf{y}_{x}=\mathbf{z}_{x}-\frac{\tilde{y}-\tilde{z}}{2}(\mathbf{y}+\mathbf{z})+\frac{\mathbf{y}_{x}^{2}-\mathbf{z}_{x}^{2}}{2(\tilde{y}-\tilde{z})}(\mathbf{y}-\mathbf{z}) .
$$

Таким образом, в формулу суперпозиции для решений уравнения (1.1) можно ввести квазилокальные переменные. Для этого согласно диаграмме (1.4) нужно положить в $(1.6)(\mathbf{u}, \mathbf{p})=1-(\tilde{u}-\tilde{p}) /(2 \mu),(\mathbf{v}, \mathbf{p})=1-(\tilde{v}-\tilde{p}) /(2 \nu)$.

\section{2. ВЕКТОРНОЕ ОБОБЩЕНИЕ УРАВНЕНИЯ ЛАНДАУ-ЛИФШИЦА}

Рассмотрим известное анизотропное (в терминологии работы [1]) векторное уравнение

$$
\mathbf{z}_{t}=D_{x}\left(\mathbf{z}_{x x}+\frac{3}{2}\left(\mathbf{z}_{x}, \mathbf{z}_{x}\right) \mathbf{z}\right)+\frac{3}{2}\langle\mathbf{z}, \mathbf{z}\rangle \mathbf{z}_{x}, \quad \mathbf{z}^{2}=(\mathbf{z}, \mathbf{z})=1 .
$$

Здесь и далее $\langle\mathbf{x}, \mathbf{y}\rangle=(\mathbf{x}, R \mathbf{y})$, где $R$ - постоянная симметричная матрица. Возможно, это уравнение интересно с точки зрения физических приложений. Как было показано Голубчиком и Соколовым [7], если $\mathbf{z} \in \mathbb{S}^{3}$, то (2.1) есть высшая симметрия уравнения Ландау-Лифшица

$$
\mathbf{z}_{t}=\mathbf{z} \times \mathbf{z}_{x x}+\mathbf{z} \times(J \mathbf{z}), \quad|\mathbf{z}|=1,
$$

где $\times$ означает векторное произведение, а матрица $J=\operatorname{diag}\left(J_{1}, J_{2}, J_{3}\right)$ описывает анизотропию. В частности, уравнение (2.2) возникает при изучении периодических и квазипериодических решений классических нелинейных волновых уравнений (уравнения синус-Гордон и нелинейного уравнения Шредингера). Решения в специальных случаях описаны в работе [8].

Полученное нами в [9] автопреобразование Беклунда уравнения (2.1) имеет вид

$$
\frac{\mathbf{y}_{x}+\mathbf{z}_{x}}{2}=\frac{\left(\mathbf{y}, \mathbf{z}_{x}\right)(\mathbf{y}+\mathbf{z})+f(\mathbf{y}+\mathbf{z}, \mu)(\mathbf{z}-(\mathbf{y}, \mathbf{z}) \mathbf{y})}{(\mathbf{y}+\mathbf{z})^{2}},
$$

где $f^{2}(\mathbf{z}, \mu)=\langle\mathbf{z}, \mathbf{z}\rangle+\mu \mathbf{z}^{2}$. В цитируемой работе мы тоже предположили коммутативность диаграммы (1.4) в случае преобразования Беклунда (2.3) и получили скалярную формулу суперпозиции для решений $\mathbf{p}, \mathbf{u}, \mathbf{v}, \mathbf{q}$ уравнения (2.1):

$$
f(\mathbf{p}+\mathbf{v}, \nu)+f(\mathbf{u}+\mathbf{p}, \mu)=f(\mathbf{q}+\mathbf{v}, \mu)+f(\mathbf{u}+\mathbf{q}, \nu) .
$$

Пусть существует преобразование Беклунда для уравнения (2.1), зависящее от квазилокальных переменных. Прямым вычислением можно проверить, что равенство

$$
f(\mathbf{y}+\mathbf{z}, \mu)=\tilde{y}-\tilde{z},
$$

где $\tilde{y}=(1 / 2) D_{x}^{-1}\left[\left(\mathbf{y}_{x}, \mathbf{y}_{x}\right)-\langle\mathbf{y}, \mathbf{y}\rangle\right], \tilde{z}=(1 / 2) D_{x}^{-1}\left[\left(\mathbf{z}_{x}, \mathbf{z}_{x}\right)-\langle\mathbf{z}, \mathbf{z}\rangle\right]$, совместно с $(2.1)$ и (2.3). Теперь из коммутативности диаграммы (1.4) и сотношений вида (2.5) для $\mathbf{p}, \mathbf{u}, \mathbf{v}, \mathbf{q}$ автоматически следует (2.4). 
С учетом (2.5) преобразование (2.3) можно переписать в виде

$$
\mathbf{y}_{x}=-\mathbf{z}_{x}+2 \frac{\left(\mathbf{y}, \mathbf{z}_{x}\right)(\mathbf{y}+\mathbf{z})+(\tilde{y}-\tilde{z})(\mathbf{z}-(\mathbf{y}, \mathbf{z}) \mathbf{y})}{(\mathbf{y}+\mathbf{z})^{2}} .
$$

Возведем последнее равенство в квадрат и выразим величину $\left(\mathbf{y}, \mathbf{z}_{x}\right)$. Подставив результат в (2.3), получаем, что (2.3) равносильно системе

$$
\begin{aligned}
\mathbf{y}_{x}+\mathbf{z}_{x} & =\frac{\mathbf{y}_{x}^{2}-\mathbf{z}_{x}^{2}}{2(\tilde{y}-\tilde{z})}(\mathbf{y}+\mathbf{z})-\frac{\tilde{y}-\tilde{z}}{2}(\mathbf{y}-\mathbf{z}), \\
\tilde{y}-\tilde{z} & =f(\mathbf{y}+\mathbf{z}, \mu) .
\end{aligned}
$$

Предположив коммутативность диаграммы (1.4) и используя соответствующие ей системы вида (2.6) для пар $\{\mathbf{u}, \mathbf{p}\},\{\mathbf{v}, \mathbf{p}\},\{\mathbf{q}, \mathbf{u}\}$ и $\{\mathbf{q}, \mathbf{v}\}$, находим формулу суперпозиции решений уравнения (2.1):

$$
\mathbf{q}=\zeta^{-1}(\mathbf{u}-\mathbf{v})^{2}\left(\xi \eta \mathbf{p}+(\mu-\nu)\left(\eta(\mathbf{u}+\mathbf{p})^{2} \mathbf{v}-\xi(\mathbf{v}+\mathbf{p})^{2} \mathbf{u}\right)\right)
$$

где

$$
\begin{gathered}
\zeta=(\mathbf{u}+\mathbf{p})^{2} \xi^{2}+(\mathbf{v}+\mathbf{p})^{2} \eta^{2}+\left(1-(\mathbf{u}+\mathbf{v}+\mathbf{p})^{2}\right) \xi \eta \\
\eta=(\tilde{u}-\tilde{v})^{2}-f^{2}(\mathbf{u}-\mathbf{v}, \mu), \quad \xi=(\tilde{u}-\tilde{v})^{2}-f^{2}(\mathbf{u}-\mathbf{v}, \nu), \\
\tilde{u}-\tilde{v}=f(\mathbf{u}+\mathbf{p}, \mu)-f(\mathbf{v}+\mathbf{p}, \nu) .
\end{gathered}
$$

\section{3. ДРУГИЕ ИНТЕГРИРУЕМЫЕ СЛУЧАИ}

Одно из представленных в работе [3] интегрируемых уравнений и его автопреобразование Беклунда записываются следующим образом:

$$
\begin{gathered}
\mathbf{z}_{t}=\mathbf{z}_{x x x}+\frac{3}{2}\left(\left(\frac{\mathbf{z}_{x}^{2}}{a}+\frac{\left(\mathbf{z}, \mathbf{z}_{x}\right)^{2}}{a\left(a-\mathbf{z}^{2}\right)}\right) \mathbf{z}\right)_{x}+\frac{3}{2}\left(\frac{\mathbf{z}_{x}^{2}}{a}+\frac{\left(\mathbf{z}, \mathbf{z}_{x}\right)^{2}}{a\left(a-\mathbf{z}^{2}\right)}\right) \mathbf{z}_{x}, \\
\mathbf{y}_{x}+\mathbf{z}_{x}=\frac{z\left(\mathbf{y}, \mathbf{z}_{x}\right)+y\left(\mathbf{z}, \mathbf{z}_{x}\right)}{z(a+y z+(\mathbf{y}, \mathbf{z}))}(\mathbf{y}+\mathbf{z})+\mu(a \mathbf{z}-(y z+(\mathbf{y}, \mathbf{z})) \mathbf{y}), \\
y^{2}=(\mathbf{y}, \mathbf{y})-a, \quad z^{2}=(\mathbf{z}, \mathbf{z})-a .
\end{gathered}
$$

Кроме того, равенство

$$
\tilde{z}-\tilde{y}=\frac{(z-y)^{2}}{\mu(a+y z+(\mathbf{y}, \mathbf{z}))},
$$

где $\tilde{y}=D_{x}^{-1}(\mathbf{y}, \mathbf{y}), \tilde{z}=D_{x}^{-1}(\mathbf{z}, \mathbf{z})$, совместно с (3.1) и $(3.2)$.

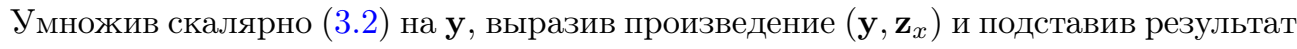
в (3.2), имеем следующее автопреобразование Беклунда:

$$
\mathbf{y}_{x}+\mathbf{z}_{x}=\frac{z\left(\mathbf{y}, \mathbf{y}_{x}\right)-y\left(\mathbf{z}, \mathbf{z}_{x}\right)}{y z(y-z)}(\mathbf{y}+\mathbf{z})-\frac{y-z}{\tilde{y}-\tilde{z}}(y \mathbf{z}+z \mathbf{y}) .
$$

По-прежнему предполагая коммутативность диаграммы (1.4), записываем систему вида (3.4), (3.3) для каждой пары решений $\{\mathbf{u}, \mathbf{p}\},\{\mathbf{v}, \mathbf{p}\},\{\mathbf{q}, \mathbf{u}\}$ и $\{\mathbf{q}, \mathbf{v}\}$. Следуя уже описанному алгоритму, получаем формулу суперпозиции для решений уравнения (3.1):

$$
\mathbf{q}=\frac{a+u v-(\mathbf{u}, \mathbf{v})}{\zeta}(\xi \eta \mathbf{p}+(\mu+\nu)(\eta[a+v p+(\mathbf{v}, \mathbf{p})] \mathbf{u}-\xi[a+u p+(\mathbf{u}, \mathbf{p})] \mathbf{v})),
$$


где

$$
\begin{aligned}
\xi= & \nu(a+v p+(\mathbf{v}, \mathbf{p}))-\mu((\mathbf{u}, \mathbf{p})+(\mathbf{u}, \mathbf{v})+u p-u v), \\
\eta= & \nu((\mathbf{v}, \mathbf{p})+(\mathbf{u}, \mathbf{v})+v p-u v)-\mu(a+u p+(\mathbf{u}, \mathbf{p})), \\
\zeta= & {[a+u p+(\mathbf{u}, \mathbf{p})] \xi^{2}+[a+v p+(\mathbf{v}, \mathbf{p})] \eta^{2}-} \\
& \quad-[a+u p+v p-u v+(\mathbf{v}, \mathbf{p})+(\mathbf{u}, \mathbf{p})+(\mathbf{u}, \mathbf{v})] \xi \eta .
\end{aligned}
$$

Скалярная формула суперпозиции для решений уравнения (3.1) имеет вид

$$
\frac{(u-p)^{2}}{\mu(a+u p+(\mathbf{u}, \mathbf{p}))}+\frac{(q-u)^{2}}{\nu(a+q u+(\mathbf{q}, \mathbf{u}))}=\frac{(v-p)^{2}}{\nu(a+v p+(\mathbf{v}, \mathbf{p}))}+\frac{(q-v)^{2}}{\mu(a+q v+(\mathbf{q}, \mathbf{v}))}
$$

Рассмотрим теперь анизотропное уравнение, представленное в [3],

$$
\mathbf{z}_{t}=\mathbf{z}_{x x x}+\frac{3}{2}\left(\left(\frac{\mathbf{z}_{x}^{2}}{a}+\frac{\left(\mathbf{z}, \mathbf{z}_{x}\right)^{2}}{a\left(a-\mathbf{z}^{2}\right)}\right) \mathbf{z}\right)_{x}+\frac{3}{2}\langle\mathbf{z}, \mathbf{z}\rangle \mathbf{z}_{x},
$$

и его автопреобразование Беклунда

$$
\begin{gathered}
\mathbf{y}_{x}+\mathbf{z}_{x}=\frac{f(a \mathbf{z}+(y z-(\mathbf{y}, \mathbf{z})) \mathbf{y})}{a-y z+(\mathbf{y}, \mathbf{z})}+\frac{z\left(\mathbf{y}, \mathbf{z}_{x}\right)-y\left(\mathbf{z}, \mathbf{z}_{x}\right)}{z(a-y z+(\mathbf{y}, \mathbf{z}))}(\mathbf{y}+\mathbf{z}), \\
f^{2}=\mu(a-y z+(\mathbf{y}, \mathbf{z}))-\langle\mathbf{y}+\mathbf{z}, \mathbf{y}+\mathbf{z}\rangle, \quad y^{2}=\mathbf{y}^{2}-a, \quad z^{2}=\mathbf{z}^{2}-a .
\end{gathered}
$$

Можно проверить, что скалярное соотношение

$$
(\tilde{y}-\tilde{z})^{2}=\mu(a-y z+(\mathbf{y}, \mathbf{z}))-\langle\mathbf{y}+\mathbf{z}, \mathbf{y}+\mathbf{z}\rangle,
$$

где

$$
\begin{aligned}
& \tilde{z}=\frac{1}{2} D_{x}^{-1}\left(\langle\mathbf{z}, \mathbf{z}\rangle+\frac{\mathbf{z}_{x}^{2}}{a}-\frac{\left(\mathbf{z}, \mathbf{z}_{x}\right)^{2}}{a z^{2}}\right), \\
& \tilde{y}=\frac{1}{2} D_{x}^{-1}\left(\langle\mathbf{y}, \mathbf{y}\rangle+\frac{\mathbf{y}_{x}^{2}}{a}-\frac{\left(\mathbf{y}, \mathbf{y}_{x}\right)^{2}}{a y^{2}}\right),
\end{aligned}
$$

совместно с (3.5) и (3.6).

С помощью алгебраических преобразований, как и в предыдущем примере, первое равенство в (3.6) можно переписать в виде

$$
\mathbf{y}_{x}+\mathbf{z}_{x}=\frac{z\left(\mathbf{y}, \mathbf{y}_{x}\right)+y\left(\mathbf{z}, \mathbf{z}_{x}\right)}{y z(y+z)}(\mathbf{y}+\mathbf{z})+\frac{\tilde{y}-\tilde{z}}{y+z}(y \mathbf{z}-z \mathbf{y}) .
$$

Формула суперпозиции для решений $\mathbf{u}, \mathbf{v}, \mathbf{p}$ и $\mathbf{q}$ уравнения (3.5) выглядит следующим образом:

$$
\mathbf{q}=\frac{a+u v-(\mathbf{u}, \mathbf{v})}{\zeta}(\xi \eta \mathbf{p}+(\mu-\nu)(\xi[a-v p+(\mathbf{v}, \mathbf{p})] \mathbf{u}-\eta[a-u p+(\mathbf{u}, \mathbf{p})] \mathbf{v})),
$$

где

$$
\begin{aligned}
\xi= & \nu(a+u v-(\mathbf{u}, \mathbf{v}))-\langle\mathbf{u}-\mathbf{v}, \mathbf{u}-\mathbf{v}\rangle-(\tilde{u}-\tilde{v})^{2} \\
\eta=\mu & (a+u v-(\mathbf{u}, \mathbf{v}))-\langle\mathbf{u}-\mathbf{v}, \mathbf{u}-\mathbf{v}\rangle-(\tilde{u}-\tilde{v})^{2} \\
\zeta=\xi^{2} & {[a-v p+(\mathbf{v}, \mathbf{p})]+\eta^{2}[a-u p+(\mathbf{u}, \mathbf{p})]-} \\
& \quad-\xi \eta[a-u p-v p-u v+(\mathbf{u}, \mathbf{p})+(\mathbf{v}, \mathbf{p})+(\mathbf{u}, \mathbf{v})] .
\end{aligned}
$$


Скалярная формула суперпозиции следует из коммутативности диаграммы (1.4) и соотношений вида (3.7) для пар решений $\{\mathbf{u}, \mathbf{p}\},\{\mathbf{v}, \mathbf{p}\},\{\mathbf{q}, \mathbf{u}\}$ и $\{\mathbf{q}, \mathbf{v}\}$ :

$$
\begin{aligned}
& \sqrt{\mu(a-u p+(\mathbf{u}, \mathbf{p}))-\langle\mathbf{u}+\mathbf{p}, \mathbf{u}+\mathbf{p}\rangle}+\sqrt{\nu(a-q u+(\mathbf{q}, \mathbf{u}))-\langle\mathbf{q}+\mathbf{u}, \mathbf{q}+\mathbf{u}\rangle}= \\
& \quad=\sqrt{\nu(a-v p+(\mathbf{v}, \mathbf{p}))-\langle\mathbf{v}+\mathbf{p}, \mathbf{v}+\mathbf{p}\rangle}+\sqrt{\mu(a-q v+(\mathbf{q}, \mathbf{v}))-\langle\mathbf{q}+\mathbf{v}, \mathbf{q}+\mathbf{v}\rangle} .
\end{aligned}
$$

Нетрудно заметить, что если в (3.5) положить $\langle\mathbf{z}, \mathbf{z}\rangle=-4(\mathbf{z}, \mathbf{z})$, сделать замену $x \rightarrow-x^{\prime}$ и перейти к пределу при $a \rightarrow \infty$, то мы получим векторное обобщение уравнения мКдФ [10]:

$$
\mathbf{z}_{t}+\mathbf{z}_{x x x}-6(\mathbf{z}, \mathbf{z}) \mathbf{z}_{x}=0 .
$$

Чтобы преобразование (3.6) перешло в правильное преобразование Беклунда для уравнения мКдФ [3], необходимо сначала выполнить подстановку $\mu=2 k^{2} / a$, а затем перейти к пределу при $a \rightarrow \infty$. В результате имеем

$$
\mathbf{y}_{x}+\mathbf{z}_{x}-(\mathbf{y}-\mathbf{z}) \sqrt{k^{2}+(\mathbf{y}+\mathbf{z})^{2}}=0,
$$

при этом равенство (3.7) примет вид

$$
(\tilde{y}-\tilde{z})^{2}=k^{2}+(\mathbf{y}+\mathbf{z})^{2},
$$

где $\tilde{y}=D_{x}^{-1} \mathbf{y}^{2}, \tilde{z}=D_{x}^{-1} \mathbf{z}^{2}$. Скалярная формула суперпозиции для решений уравнения (3.5) дает соответствующую формулу для векторного обобщения уравнения мКд $\Phi$, представленную в [5],

$$
\sqrt{k_{1}^{2}+(\mathbf{u}+\mathbf{p})^{2}}+\sqrt{k_{2}^{2}+(\mathbf{q}+\mathbf{u})^{2}}=\sqrt{k_{2}^{2}+(\mathbf{v}+\mathbf{p})^{2}}+\sqrt{k_{1}^{2}+(\mathbf{q}+\mathbf{v})^{2}},
$$

а векторная формула суперпозиции в силу (3.11) запишется несколько иначе (ср. с работой [5]):

$$
\mathbf{q}=\mathbf{p}+\frac{\left(k_{2}^{2}-k_{1}^{2}\right)(\mathbf{u}-\mathbf{v})}{(\tilde{u}-\tilde{v})^{2}-(\mathbf{u}-\mathbf{v})^{2}}
$$

где $k_{1}, k_{2}-$ параметры.

\section{ЗАКЛЮЧИТЕЛЬНЫЕ ЗАМЕЧАНИЯ}

Достоверность всех формул суперпозиции мы проверили прямым расчетом на компьютере. Для демонстрации этапов процесса проверки рассмотрим (3.12).

Поскольку $\mathbf{p}, \mathbf{u}, \mathbf{v}$ и $\mathbf{q}$ - решения уравнения (3.10), вычислим производную (3.12) по $t$ с учетом (3.10). Имеем

$$
-\mathbf{q}_{x x x}+6 \mathbf{q}^{2} \mathbf{q}_{x}+\mathbf{p}_{x x x}-6 \mathbf{p}^{2} \mathbf{p}_{x}-f_{t}(\mathbf{u}-\mathbf{v})-f\left(\mathbf{u}_{x x x}-6 \mathbf{u}^{2} \mathbf{u}_{x}-\mathbf{v}_{x x x}+6 \mathbf{v}^{2} \mathbf{v}_{x}\right)=0,
$$

где здесь и далее $f=\left(k_{2}^{2}-k_{1}^{2}\right)\left((\tilde{u}-\tilde{v})^{2}-(\mathbf{u}-\mathbf{v})^{2}\right)^{-1}$. Исключим из (3.13) вектор q, все его производные по $x$ и скалярное произведение (q, q) с помощью (3.12), получим

$$
\begin{aligned}
\mathbf{p}_{x x x} & +f_{x x x}(\mathbf{u}-\mathbf{v})+f\left(\mathbf{u}_{x x x}-\mathbf{v}_{x x x}\right)+3 f_{x x}\left(\mathbf{u}_{x}-\mathbf{v}_{x}\right)+3 f_{x}\left(\mathbf{u}_{x x}-\mathbf{v}_{x x}\right)- \\
& -6\left(\mathbf{p}^{2}+2 f((\mathbf{u}, \mathbf{p})-(\mathbf{v}, \mathbf{p}))+f^{2}\left(\mathbf{u}^{2}-2(\mathbf{u}, \mathbf{v})+\mathbf{v}^{2}\right)\right) \times \\
& \quad \times\left(\mathbf{p}_{x}+f_{x}(\mathbf{u}-\mathbf{v})+f\left(\mathbf{u}_{x}-\mathbf{v}_{x}\right)\right)- \\
& -\left(\mathbf{p}_{x x x}-6 \mathbf{p}^{2} \mathbf{p}_{x}-f_{t}(\mathbf{u}-\mathbf{v})-f\left(\mathbf{u}_{x x x}-6 \mathbf{u}^{2} \mathbf{u}_{x}-\mathbf{v}_{x x x}+6 \mathbf{v}^{2} \mathbf{v}_{x}\right)\right)=0 .
\end{aligned}
$$


Векторы $\mathbf{u}_{x x}, \mathbf{v}_{x x}, \mathbf{u}_{x}, \mathbf{v}_{x}$ в последнем соотношении заменим на соответствующие выражения, используя преобразования Беклунда:

$$
\begin{array}{ll}
\mathbf{u}_{x}=-\mathbf{p}_{x}+(\mathbf{u}-\mathbf{p})(\tilde{u}-\tilde{p})=0, & \tilde{u}-\tilde{p}=\sqrt{k_{1}^{2}+(\mathbf{u}+\mathbf{p})^{2}} \\
\mathbf{v}_{x}=-\mathbf{p}_{x}+(\mathbf{v}-\mathbf{p})(\tilde{v}-\tilde{p})=0, & \tilde{v}-\tilde{p}=\sqrt{k_{2}^{2}+(\mathbf{u}+\mathbf{p})^{2}}
\end{array}
$$

Таким образом, уравнение (3.13) приводится к уравнению, не содержащему ни вектор q, ни его производные по $x$, ни какие-либо их скалярные произведения. При этом мы учли только уравнение (3.10) и соотношения (3.12)-(3.14), никак не используя коммутативность диаграммы (1.4). То есть предположение о коммутативности диаграммы (1.4) необходимо только для построения формулы суперпозиции. Легко заметить, что члены, содержащие $\mathbf{p}_{x x x}$ и $\mathbf{p}_{x x}$, сокращаются. Упрощая с помощью (3.14) выражения при $\mathbf{p}_{x}, \mathbf{u}$ и $\mathbf{v}$, мы установили, что (3.13) обращается в тождество.

Мы рассмотрели лишь малую часть представленных в [1]-[4] интегрируемых векторных уравнений. Остальные уравнения (и их преобразования Беклунда) записываются достаточно громоздко, поэтому для их исследования в контексте данной работы нужны убедительные мотивы. Отметим только, что в результате уже проведенных расчетов мы можем сделать следующие выводы о свойствах автопреобразований Беклунда векторных уравнений.

1. Каждое векторное преобразование Беклунда $\mathbb{B}(\mathbf{u}, \mathbf{v} ; \mu)$ имеет совместную с ним скалярную составляющую, зависящую от квазилокальных переменных.

2. Для построения векторной формулы суперпозиции всегда требуется привести векторную часть преобразования Беклунда к такому виду, чтобы $\mathbb{B}(\mathbf{u}, \mathbf{v} ; \mu) \equiv$ $\mathbb{B}(\mathbf{v}, \mathbf{u} ; \mu)$.

Благодарности. Автор благодарит В. В. Соколова и В. Е. Адлера за полезные обсуждения. Особая признательность А. Г. Мешкову за наставления и постоянную поддержку. Работа поддержана Министерством образования РФ (проект № 1.5.07).

\section{Список литературы}

[1] A. G. Meshkov, V. V. Sokolov, Comm. Math. Phys., 232:1 (2002), 1-18.

[2] А. Г. Мешков, В. В. Соколов, ТМФ, 139 (2004), 192-208.

[3] М. Ю. Балахнев, ТМФ, 142 (2005), 13-20.

[4] A. G. Meshkov, M. Ju. Balakhnev, SIGMA, 1 (2005), 027; arXiv: nlin.SI/0512032.

[5] М. Ю. Балахнев, Матем. заметки, 82:4 (2007), 501-503.

[6] L. Bianchi, Lezioni di Geometria Differenziale. Seconda edizione, riveduta e cosiderevolmente aumentata, in due volumi, V. II, Enrico Spoerri, Pisa, 1903.

[7] И. З. Голубчик, В. В. Соколов, ТМФ, 124 (2000), 62-71.

[8] E. K. Sklyanin, On complete integrability of the Landau-Lifshitz equation, Preprint E-3-79, Lomi, Leningrad, 1979.

[9] M. Ju. Balakhnev, Appl. Math. Lett., 18:12 (2005), 1363-1372.

[10] С. И. Свинолупов, В. В. Соколов, ТМФ, 100:2 (1994), 214-218. 